\title{
Determination of the polymerisation rate of a low-toxicity diacetone acrylamide-based holographic photopolymer using Raman spectroscopy
}

\author{
Dervil Cody \\ Technological University Dublin, dervil.cody@tudublin.ie \\ Emilia Mihaylova \\ Technological University Dublin, emilia.mihaylova@tudublin.ie \\ Luke O'Neill \\ FOCAS Research Institute, Technological University Dublin, Luke.oneill@tudublin.ie
}

See next page for additional authors

Follow this and additional works at: https://arrow.tudublin.ie/scschphyart

Part of the Materials Science and Engineering Commons, Optics Commons, and the Polymer Chemistry Commons

\section{Recommended Citation}

Cody, D., Mihaylove, E. \& O'Neill, Luke. (2016). Determination of the polymerisation rate of a low-toxicity diacetone acrylamide-based holographic photopolymer using Raman spectroscopy. Optical Materials, vol. 48, Oct. 2015, pp. 12-17. doi.org/10.1016/j.optmat.2015.07.016

This Article is brought to you for free and open access by the School of Physics \& Clinical \& Optometric Science at ARROW@TU Dublin. It has been accepted for inclusion in Articles by an authorized administrator of ARROW@TU Dublin. For more information, please contact arrow.admin@tudublin.ie, aisling.coyne@tudublin.ie, gerard.connolly@tudublin.ie.

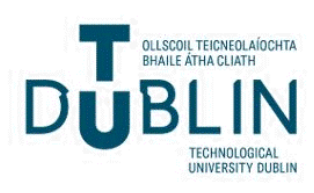




\section{Authors}

Dervil Cody, Emilia Mihaylova, Luke O'Neill, and Izabela Naydenova 


\title{
Determination of the polymerisation rate of a low-toxicity diacetone acrylamide-based holographic photopolymer using Raman spectroscopy
}

\author{
D. Cody ${ }^{\mathrm{a}, \mathrm{b}}$, E. Mihaylova ${ }^{\mathrm{a}, 1}$, L. O’Neill ${ }^{\mathrm{b}, \mathrm{c}}$, I. Naydenova $^{\mathrm{a}, \mathrm{b}, *}$ \\ ${ }^{a}$ Centre for Industrial and Engineering Optics, Dublin Institute of Technology, Dublin 8, Ireland \\ ${ }^{\mathrm{b}}$ School of Physics, College of Sciences and Health, Dublin Institute of Technology, Dublin 8, Ireland \\ ${ }^{\mathrm{c}}$ FOCAS Research Institute, Dublin Institute of Technology, Dublin 8, Ireland
}

\section{A R T I C L E I N F O}

\section{Article history:}

Received 14 April 2015

Received in revised form 9 July 2015

Accepted 10 July 2015

Available online 17 July 2015

\section{Keywords:}

Photopolymers

Holographic recording materials

Polymerisation rate

Acrylamide-based photopolymer

Studies of polymerisation rates by Raman

spectroscopy

\begin{abstract}
A B S T R A C T
The polymerisation rate of a low-toxicity Diacetone Acrylamide (DA)-based photopolymer has been measured for the first time using Raman spectroscopy. A value for the polymerisation rate of $0.020 \mathrm{~s}^{-1}$ has been obtained for the DA photopolymer by modelling the polymerisation reaction dynamics as a stretched exponential or Kohlrausch decay function. This is significantly lower than the polymerisation rate of $0.100 \mathrm{~s}^{-1}$ measured for the well known Acrylamide (AA)-based photopolymer composition. The effect of the additive glycerol on the polymerisation rate of the DA-based photopolymer has also been investigated. The inclusion of glycerol is observed to increase the rate of polymerisation of the DA photopolymer by up to $60 \%$. It is also observed that the polymerisation rate of the DA photopolymer is less dependent on the recording intensity when glycerol is present.
\end{abstract}

ㄷ 2015 Elsevier B.V. All rights reserved.

\section{Introduction}

The use of photopolymer media for holographic recording is well documented. Photopolymers have many properties including high sensitivity, large dynamic range, and a self-processing nature, which make them suitable for a wide range of holography-based applications. Among these, some of the most popular applications include holographic optical elements, holograms for security and authentication, and holographic sensors. Acrylamide (AA)-based photopolymers are one of the most widely used water-soluble photopolymers for development of holographic applications such as those listed above, as demonstrated by the huge volume of literature and patents available on their development and use. This is easy to understand, as they are water soluble, easy to prepare, low cost and readily record bright, efficient holograms. In recent years however, as holographic applications are becoming more and more of a reality, there has been a move in the research

\footnotetext{
* Corresponding author at: Centre for Industrial and Engineering Optics/School of Physics, Dublin Institute of Technology, Dublin 8, Ireland. E-mail address: izabela.naydenova@dit.ie (I. Naydenova).

1 Current affiliation: Department of Mathematics, Informatics and Physics, Agricultural University, Plovdiv, Bulgaria.
}

community towards photopolymer materials that not only perform well holographically, but that are low-toxicity and environmentally compatible.

The authors have previously reported on the development of a novel photopolymer composition which uses the non-toxic monomer Diacetone Acrylamide (DA) as a replacement for AA [1-3]. This has been done to reduce the potential occupational and environmental hazards involved in future large-scale material development and device fabrication. The new material's holographic recording capability has previously been shown to surpass that of other reported low-toxicity photopolymer alternatives [4-7].

Aside from reduced toxicity, the DA monomer differs primarily from the AA monomer in terms of its structure. The size of the DA monomer molecule $(10 \times 4 \AA)$ is nearly twice that of the AA monomer (approximate size of $5 \times 4 \AA$ ). Theoretically this may be seen as a disadvantage, as reduced monomer mobility during holographic recording would be expected to limit grating growth. However, diffraction efficiency values for transmission gratings of up to $80 \%$ have been observed in $60 \mu \mathrm{m}$ thick photopolymer layers, corresponding to refractive index modulation values of up to $3.3 \times 10^{-3}$. In addition, the larger size of the DA monomer has previously been shown to be beneficial for the development of nanocomposite polymers containing porous BEA-structure zeolite nanoparticles [8]. 
The DA photopolymer has shown itself to be a suitable material for optical sensing devices, for a range of analytes. With the inclusion of zeolite nanoparticles it is effective as a gas sensor for different molecules, including hydrocarbons [9]. It has also been demonstrated that DA photopolymer-based holographic gratings can operate as reversible sensors for relative humidity (RH) and temperature [10].

In order to have a full picture of the holographic recording capabilities of any material, and therefore it's suitability for different holographic applications, it is necessary to be informed of the key factors which govern hologram formation in the medium, namely the rates of polymerisation and monomer diffusion. Here, the experimental measurement of the polymerisation rate of a DA-based photopolymer is reported for the first time.

The polymerisation rate of the AA photopolymer has previously been measured [11]. It is important to experimentally quantify the rate of polymerisation of the DA photopolymer in order to identify the optimum holographic recording conditions including recording intensity and exposure energy, thereby allowing the new material's potential to be completely utilised. The effect of the inclusion of the plasticizer glycerol on the rate of polymerisation of the DA photopolymer is described, allowing further insight into its role within the photopolymer. Comparison is also made with previously reported data for the AA photopolymer, which provides data on the effect of monomer size on the rate of polymerisation.

\section{Theory}

The polymerisation rate of a material is the rate at which the individual monomer molecules are polymerised, forming polymer chains. One of the key factors that affects the dynamics of holographic recording in photopolymer materials is the ratio of the rate of polymerisation to the rate of diffusion in the material, which is a high interest research area, and various theories have been proposed to explain this relationship [12-23].

The Two-Way Diffusion model was first proposed by Naydenova et al. and Martin et al. to explain the observed relationship between the polymerisation and diffusion rates in AA-based photopolymer formulations [12,13]. It was then fully developed as a mathematical model by Babeva et al. [14]. During holographic recording, the monomer molecules diffuse into and within the illuminated regions, encounter a free radical, and become polymerised. If the polymerisation reaction occurs at too fast a rate, a proportion of the monomer molecules will not diffuse quickly enough before the permeability of the bright fringe region decreases significantly and hence cannot be polymerised in this region, and so the refractive index modulation, $\Delta n$, of the recorded hologram will be reduced accordingly. The length of the polymer chains formed is also dependent on the recording intensity used. At high recording intensities short polymer chains are formed due to the increased concentration of initiating species, which may diffuse out of the illuminated regions, thus degrading $\Delta n$ of the recorded grating. Alternatively, the Non-local PhotoPolymerization Driven Diffusion (NPDD) Model [22,23] assumes that the chains grow away from their initiation point resulting in "spreading" of the polymer. This model predicts that improvement at high spatial frequencies can be achieved if shorter polymer chains are created during holographic recording [23].

The dependence of the rate of polymerisation on recording intensity has previously been investigated. Some early models [24-26] predict a linear dependence on intensity. Kwon et al. postulated that the polymerisation rate depends on the square root of the recording intensity [27] and define the relationship between polymerisation rate and recording intensity as: $\frac{1}{t}=k I^{0.5}$

where $t$ is the polymerisation time constant, $k$ is the polymerisation rate, and $I$ is the recording intensity. However, these models assume that the rate of initiation is equal to the rate of termination, and that the free radical concentration is constant throughout polymerisation. Jallapuram et al. [11] adapted the above equation for the more general form:

$\frac{1}{t}=k I^{\gamma}$

where $\gamma$ is the polymerisation rate dependence parameter, which determines the dependence on recording intensity.

A Raman spectroscopy method previously developed by Jallapuram et al. [11] has been employed here to determine for the first time the polymerisation rate of the DA-based photopolymer. In a Raman spectrometer, laser light of a defined wavelength is incident on a material which excites the molecules to produce molecular vibrations, phonons or other excitations in the system. This excitation results in the energy of the laser photons being shifted up or down, which provides information about the vibrational modes in the system. Vibrational information is specific to the chemical bonds and symmetry of molecules [28]. Jallapuram et al. determined the polymerisation rate of the AA photopolymer by measuring the real-time change in the intensity of the Raman peaks at 1607 and $1629 \mathrm{~cm}^{-1}$ [11] within the photopolymer as it was polymerised for different exposure times. These peaks correspond to the $\mathrm{C}=\mathrm{C}$ double bonds of the $\mathrm{AA}$ monomer and the crosslinking monomer bisacrylamide (BA) respectively. The experiments were carried out for uniform illumination, which ensures that the polymerisation rate does not depend on an assumed diffusion time. The polymerisation rates for the AA and BA monomers were measured to be $0.100 \mathrm{~s}^{-1}$ and $0.114 \mathrm{~s}^{-1}$ respectively. The polymerisation rate dependence parameter $\gamma$ was found to be 0.27 and 0.32 for the AA and BA monomers. This differs from the value for $\gamma$ assumed by Kwon et al. of 0.5 , and implies that the relationship between the polymerisation rate and recording intensity is weaker for the AA photopolymer system than expected.

This method also allows for investigation for the first time of the influence of the plasticizer Glycerol on the polymerisation rate and the polymerisation rate dependence parameter of the DA photopolymer. The effect of glycerol on the holographic recording ability of the DA photopolymer in the transmission mode of recording has previously been investigated [2]. A 30\% decrease in the maximum achievable $\Delta n$ of the DA-based photopolymer was observed at $1000 \mathrm{l} / \mathrm{mm}$ with the inclusion of glycerol; however, a more uniform response to recording intensity is observed and $\Delta n$ values of up to $2.25 \times 10^{-3}$ are still achievable. The increased uniformity of the response to recording intensity is attributed to glycerol's nature as a plasticiser, resulting in increased diffusion of monomer molecules, and is a desirable feature for large scale production. It has been demonstrated that glycerol also significantly improves the optical quality, uniformity and reproducibility of the DA photopolymer layers. This is very important for any future device fabrication. Further information about the effect of glycerol on the holographic recording mechanisms of the DA photopolymer system can therefore be obtained.

\section{Experimental}

\subsection{Materials}

The DA0 (without glycerol) and DAG (with glycerol) photopolymer compositions were prepared as described elsewhere [2]. 
Samples were prepared by depositing $0.5 \mathrm{ml}$ of photopolymer solution on to $75 \times 25 \mathrm{~mm}$ glass microscope slides, and allowed to dry for $12-24 \mathrm{~h}$ in darkness under normal laboratory conditions $\left(20-25^{\circ} \mathrm{C}, 40-60 \% \mathrm{RH}\right)$. Layer thickness was measured using a white-light surface profiler (Micro XAM S/N 8038), and was found to be $60 \pm 5 \mu \mathrm{m}$.

\subsection{Methods}

\subsubsection{Sample polymerisation method}

The experimental setup used for exposing the DA0 and DAG samples is shown in Fig. 1. DA0 and DAG photopolymer layers were exposed to a single homogeneous $532 \mathrm{~nm}$ vertically polarized beam at intensities of $0.5,2$ and $4 \mathrm{~mW} / \mathrm{cm}^{2}$. Exposure times of 5 , $10,15,25,50,100,200,400$ and $800 \mathrm{~s}$ were used. Unsensitised layers of the same thickness were prepared also for reference, as no polymerisation would take place in these layers. On exposure to light, photoinduced polymerisation occurs within the sample and the $\mathrm{C}=\mathrm{C}$ double bonds of the monomer are converted to $\mathrm{C}-\mathrm{C}$ single bonds.

\subsubsection{Raman spectroscopy method}

A Horiba Jobin Yvon LabRAM HR 800 Raman spectrometer was used to obtain the Raman spectra of the exposed area of each sample. A $10 \mathrm{~mW} / \mathrm{cm}^{2} 659.27 \mathrm{~nm}$ laser was used to take the spectra as the Erythrosin B-sensitised samples have negligible absorbance at this wavelength. An $1800 \mathrm{l} / \mathrm{mm}$ grating and a $100 \times$ objective were used. Ten $100 \mathrm{~s}$ exposures were accumulated and averaged for each sample.

The polymerisation rate of the DA-based photopolymer was determined from the Raman spectra by monitoring the change in intensity of the $1628 \mathrm{~cm}^{-1}$ peak as the material is polymerised. This peak is in fact the superposition of two separate peaks at 1624.7 and $1629.9 \mathrm{~cm}^{-1}$ which correspond to the $\mathrm{C}=\mathrm{C}$ double bonds of the DA and BA monomers respectively as shown in Fig. 2. The intensity of the $1628 \mathrm{~cm}^{-1}$ peak in these samples was monitored with respect to a reference double peak at $1450 \mathrm{~cm}^{-1}$, the intensity of which remained constant during polymerisation. An example of the Raman spectra obtained for DA photopolymer samples exposed to a recording intensity of $4 \mathrm{~mW} / \mathrm{cm}^{2}$ for different durations is shown in Fig. 3.

Due to the restrictions imposed by the resolution of the Raman spectrometer, it was not possible to resolve the $C=C$ peaks of the DA and BA monomer molecules separately within the photopolymer spectrum, therefore the polymerisation rates of the DA and BA monomers could not be individually calculated for the DA-based photopolymer composition. However, by monitoring the change in the intensity of the superimposed peak, the polymerisation rate of the overall composition can be determined. Unfortunately, this does not allow for direct comparison of the DA-based photopolymers with the standard AA photopolymer, for as discussed above, the $C=C$ double bond peaks for the $A A$ and $\mathrm{BA}$ monomers were resolvable in this instance.

\subsubsection{Data analysis method}

A single exponential decay function was used by Jallapuram et al. to fit the intensity vs. exposure time data for the AA-based

\section{$532 \mathrm{~nm}$ YAG Laser}

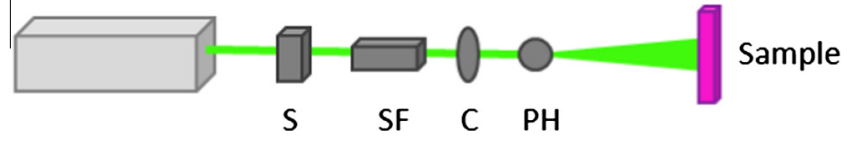

Fig. 1. Experimental setup for exposing the Erythrosin B-sensitised DA0 and DAG samples. S: shutter, SF: spatial filter, C: collimator, PH: pinhole.

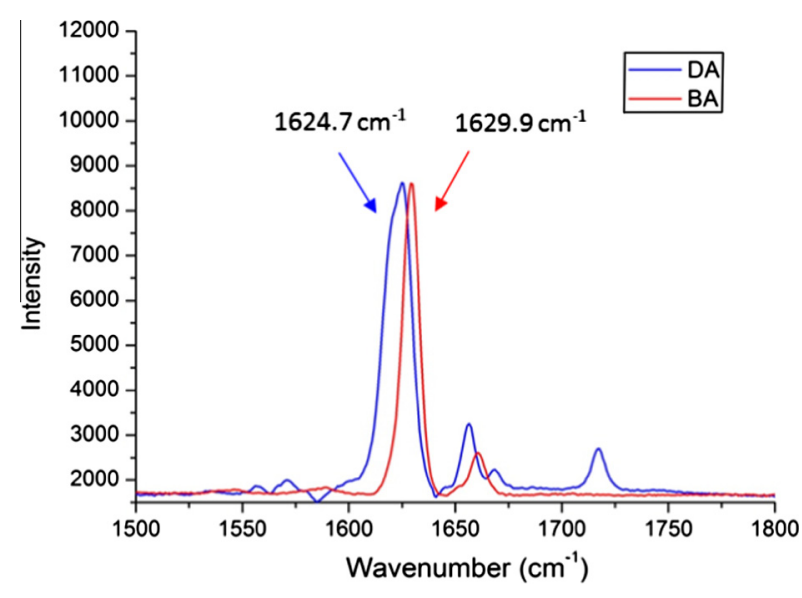

Fig. 2. Raman spectra taken at an excitation wavelength of $659.27 \mathrm{~nm}$ of the DA and BA monomers. The peaks at $1624.7 \mathrm{~cm}^{-1}$ (blue) and $1629.9 \mathrm{~cm}^{-1}$ (red) correspond to the $\mathrm{C}=\mathrm{C}$ double bonds of $\mathrm{DA}$ and $\mathrm{BA}$ respectively. (For interpretation of the references to colour in this figure legend, the reader is referred to the web version of this article.)

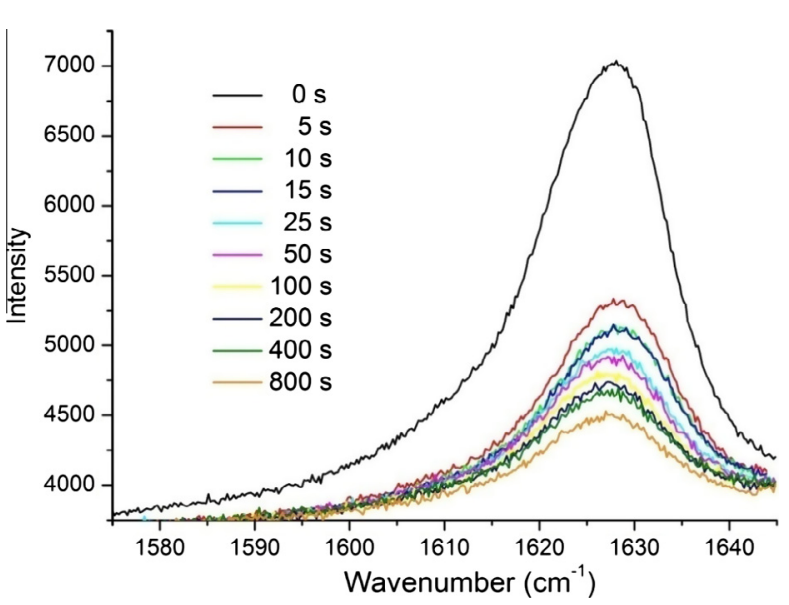

Fig. 3. Raman spectra taken at an excitation wavelength of $659.27 \mathrm{~nm}$ of DAO photopolymer samples exposed to a beam intensity of $4 \mathrm{~mW} / \mathrm{cm}^{2}$ for durations of 0 , $5,10,15,25,50,100,200,400$ and $800 \mathrm{~s}$.

photopolymer as discussed in [11]. Different exponential decay functions including single, double and triple exponential decays, were fitted to the intensity vs. exposure time data for both the DAO and DAG data. However, none of these functions gave a good fit to the data obtained. The function best observed to fit the data was the Kohlrausch stretched exponential function of the form:

$y(x)=y_{0}+A e^{\left(-\frac{x}{t}\right)^{\beta}}$

where $\beta$ is some perturbation of the system for which, $0<\beta<1, t$ is the time constant for the reaction, $y_{0}$ is the baseline and $A$ is an amplitude factor.

The Krohlrausch function, or stretched exponential function as it is now more commonly known, was first described by Rudolph Krohlrausch in 1854 [29-31]. Krohlrausch found that this function accurately describes the time evolution of the discharge of a capacitor, for which standard exponential functions did not provide a good fit. It has since been observed that the stretched exponential function is often more appropriate for modelling relaxation processes in different materials, including polymers, than standard exponentials [30]. This is partly due to the fact that a relaxation process depends on the entire spectrum of relaxation times, so its structure will be non-linear and not purely exponential. 
De Gennes uses the stretched exponential function to model large-scale chain dynamics of polydispersive systems [32]. He defines $\beta$ as an exponent that is sensitive to the presence or absence of entanglement of the polymer chains. Chen postulates that $\beta$ may be related to the distribution of chain lengths, or disorder of the system [33]. As the value for $\beta$ goes to 1 , a normal exponential is obtained which produces a single time constant for the reaction. Alternatively, as the value for $\beta$ goes to 0 , multiple time constants are necessary, implying more disorder in the system.

\section{Results}

\subsection{Polymerisation rate calculation for DAO and DAG photopolymers}

The intensity of the $1628 \mathrm{~cm}^{-1}$ peak was plotted vs. exposure time for the DA0 and DAG photopolymer layers when exposed to recording intensities of $0.5,2$ and $4 \mathrm{~mW} / \mathrm{cm}^{2}$. The data obtained is given in Fig. 4. The data was fitted with the stretched exponential decay function given by Eq. (3) using Origin v. 8.5 software. The values for the time constant, $t$, and $\beta$ were extracted for both the DA0 and DAG compositions, and the results are shown in Table 1 . The $R^{2}$ values for each set of data show good agreement between the stretched exponential fit and the experimental data. As the recording intensity is increased, the time constant is reduced because the polymerisation reaction occurs at a faster rate. Due to this it is observed that the uncertainty on the time constant $t$ becomes relatively larger. The values of $\gamma$ and $k$ were determined using Eq. (2) by first plotting the logarithm of the polymerisation time constant $t$ against the logarithm of the exposure
Table 1

Data from stretched exponential function fit of intensity vs. exposure time.

\begin{tabular}{lllll}
\hline & Intensity $\left(\mathrm{mW} / \mathrm{cm}^{2}\right)$ & $t(\mathrm{~s})$ & $\beta$ & $R^{2}$ value \\
\hline \multirow{2}{*}{ DA0 } & 0.5 & 100.05 & 0.199 & 0.98 \\
& 2 & 25.28 & 0.155 & 0.99 \\
& 4 & 10.40 & 0.153 & 0.99 \\
DAG & 0.5 & 53.41 & 0.449 & 0.99 \\
& 2 & 16.57 & 0.214 & 0.99 \\
& 4 & 13.77 & 0.201 & 0.99
\end{tabular}

intensity, and then fitting the data using a linear function as shown in Fig. 5. The slope of the linear fit equals $-\gamma$, and the intercept of the linear fit is equal to $-\log (k)$. This produces values for the polymerisation rate of $k=0.020 \pm 0.008 \mathrm{~s}^{-1}$ for DA0 and $k=0.032 \pm 0.005 \mathrm{~s}^{-1}$ for DAG. Values for the polymerisation rate dependence parameter of $1.03 \pm 0.06$ and $0.78 \pm 0.09$ were obtained for DA0 and DAG respectively.

\section{Discussion}

\subsection{Comparison of the DAO and DAG polymerisation rates, $k$}

The rates of polymerisation for the DA0 and DAG photopolymer were found to be $0.020 \mathrm{~s}^{-1}$ and $0.032 \mathrm{~s}^{-1}$ respectively. This implies that the inclusion of glycerol increases the rate of polymerisation of the DA photopolymer by as much as $60 \%$. As discussed previously, glycerol is a plasticiser. Therefore, it is expected that its inclusion improves the ability of the unreacted DA monomer
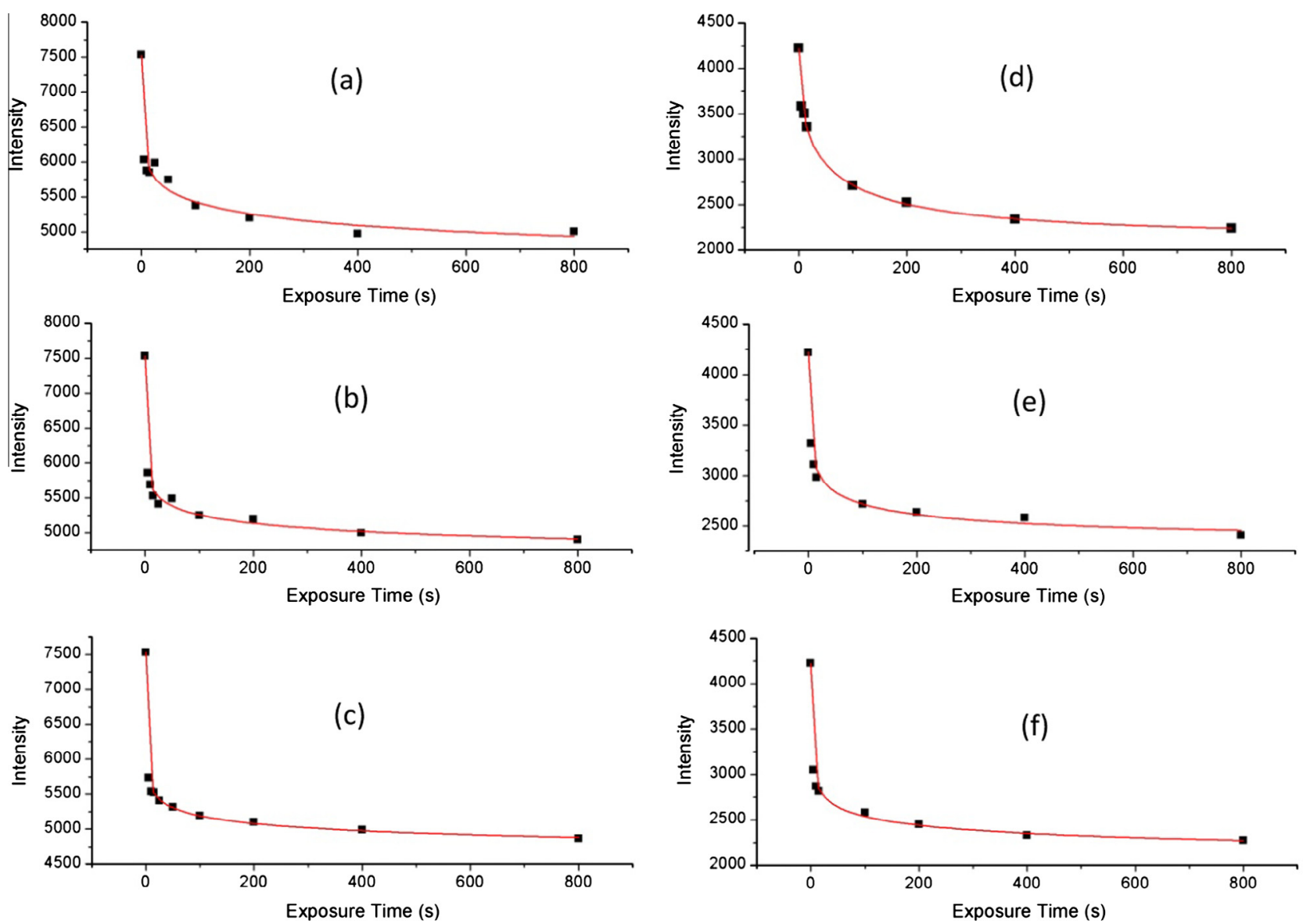

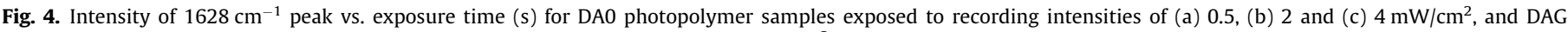
photopolymer samples exposed to recording intensities of (d) 0.5 , (e) 2 and (f) $4 \mathrm{~mW} / \mathrm{cm}^{2}$. All data is fitted with the Kohlrausch function. 


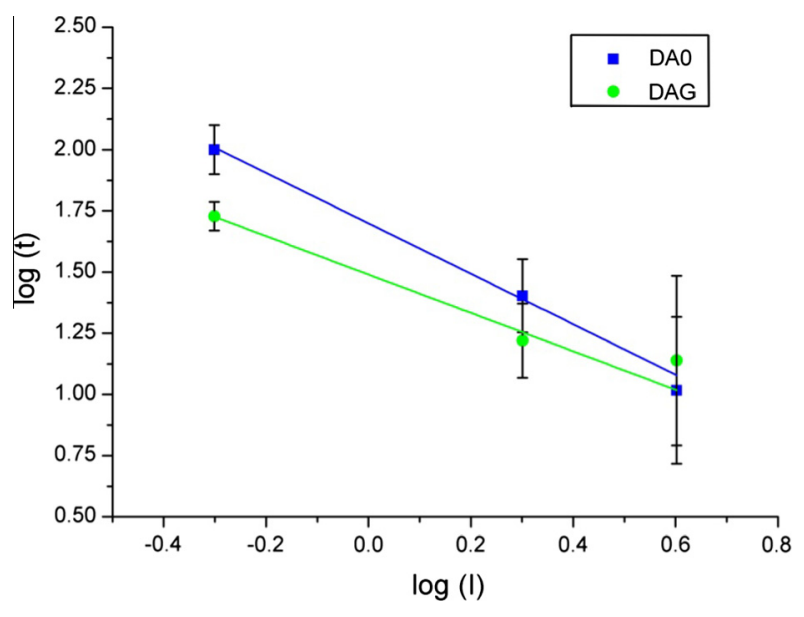

Fig. 5. $\log (t)$ vs. $\log (I)$ for the DAO and DAG photopolymers.

molecules, and also the free radicals, to diffuse within the photopolymer layer during illumination. This increases the probability of an interaction which will result in the initiation of polymer chains or growth of existing chains, thus increasing the rate of polymerisation of the DA photopolymer.

The rate of polymerisation of the AA-based photopolymer was calculated by Jallapuram et al. to be $0.100 \mathrm{~s}^{-1}$ [11]. As mentioned previously, direct comparison cannot be made between the DA-based and AA-based material due to the inability to resolve the separate $\mathrm{C}=\mathrm{C}$ bonds of the DA and BA monomers within the photopolymer. However, it is likely that the polymerisation rate of the AA-based formulation is faster than for the DA-based compositions due to the smaller size of the AA monomer molecules, allowing for faster diffusion of the monomer molecules within the photopolymer layer. This is reflected in the values for the polymerisation rates obtained for DA0 and DAG. It is worth noting that the difference in the rates will be observed only in the case of a diffusion rate-limited polymerisation (the polymerisation rate is much faster than the diffusion rate).

\subsection{Comparison of the DAO and DAG polymerisation rate dependence on intensity (parameter $\gamma$ )}

The polymerisation rate intensity dependence parameter $\gamma$ was found to be 1.03 and 0.78 for the DAO and DAG photopolymers respectively. $\gamma$ is a direct measure of the dependence of the polymerisation rate on the recording intensity used. The value for $\gamma$ for DAG is $24 \%$ lower than for DA0. Therefore, the response of the DAG photopolymer is less dependent on recording intensity than the response of the DA0 photopolymer. This agrees with the results observed in [2], which showed that the $\Delta n$ of transmission gratings recorded in the DAG photopolymer had limited intensity dependence in comparison to that of the DA0 photopolymer. In principle, increasing the recording intensity increases the concentration of free radicals produced, and therefore increases the probability of a free radial reacting with a monomer molecule to initiate a polymer chain. However, the probability of this reaction occurring is already increased due to the inclusion of glycerol and increasing the permeability of the polymer, as discussed above. Therefore, the dependence of polymerisation rate on recording intensity within the studied intensity range is limited for DAG.

\subsection{Comparison of the DAO and DAG $\beta$ values}

Values for $\beta$ were extracted from the stretched exponential fits for both the DAO and DAG photopolymer. As discussed

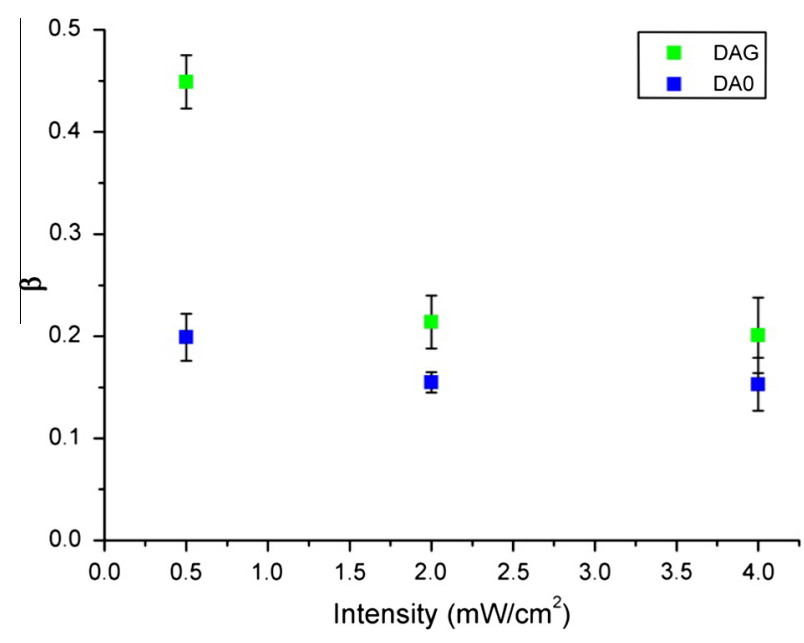

Fig. 6. $\beta$ vs. intensity $\left(\mathrm{mW} / \mathrm{cm}^{2}\right)$ for DAO and DAG photopolymers.

earlier, it has been postulated by De Gennes that $\beta$ is sensitive to the presence or absence of entanglement of the polymer chains [32]. As $\beta$ approaches unity, a normal exponential is realised, implying an ordered system. It can therefore be inferred that as $\beta$ approaches zero, the system moves away from a normal exponential, resulting in an increase in the level of disorder of the system. The values for $\beta$ as a function of recording intensity for both the DA0 and DAG compositions are shown in Fig. 6 . It is seen that as the recording intensity increases, the value for $\beta$ decreases. This is expected, as by increasing the recording intensity, the number of free radicals generated is increased, resulting in an increase in the number of growing polymer chains. Therefore, the extent of chain entanglement, or disorder of the system, is increased. At all recording intensities, the value for $\beta$ is higher for the DAG composition than for DA0. This implies that the inclusion of glycerol in the DA photopolymer composition decreases the degree of entanglement and thus the level of disorder of the growing polymer chains. The difference in $\beta$ between DAO and DAG is more significant at lower recording intensities. Glycerol is a plasticiser and therefore may be promoting increased diffusion of monomer molecules as well as the growing polymer chains, resulting in increased homogeneity of the chain lengths. This may result in less disorder in the system as postulated by Chen [33]. As the recording intensity is increased, this effect becomes less pronounced due to the increased concentration of free radicals.

\section{Conclusions}

The polymerisation rates of the DA0 and DAG photopolymers have been measured experimentally for the first time using Raman spectroscopy methods. A stretched exponential decay function has been applied to the data to extract polymerisation rate values of $0.020 \pm 0.008 \mathrm{~s}^{-1}$ and $0.032 \pm 0.005 \mathrm{~s}^{-1}$ for DA0 and DAG respectively. This implies that the inclusion of glycerol increases the rate of polymerisation of the DA photopolymer by up to $60 \%$. The faster polymerisation rate observed for DAG due to the inclusion of the plasticiser glycerol, allowing for increased diffusion of the monomer molecules and initiating species thereby increasing the rate at which the polymerisation reaction can occur.

The polymerisation rate dependence parameter $\gamma$ was found to be $1.03 \pm 0.06$ and $0.78 \pm 0.09$ for the DA0 and DAG photopolymers respectively. The lower $\gamma$ value for DAG reflects the lesser dependence of the polymerisation rate on the recording intensity used for the glycerol-doped material. 
By studying the values for the parameter $\beta$ extracted from the stretched exponential fit for the DA0 and DAG compositions, new information has been provided about the role of glycerol in the DA photopolymer. The results indicate that glycerol increases the homogeneity of the DA polymer chain length distribution, and thereby reduces the level of disorder in the system. It has also been observed that the inclusion of glycerol in the DA photopolymer is beneficial for holographic recording in reflection mode. This will be discussed further in a future publication.

\section{References}

[1] D. Cody, I. Naydenova, E. Mihaylova, New non-toxic holographic photopolymer, J. Opt. 14 (2012) 015601.

[2] D. Cody, I. Naydenova, E. Mihaylova, Effect of glycerol on a diacetone-acrylamide based holographic photopolymer, Appl. Opt. 52 (3) (2013) 489-494.

[3] D. Cody, A. Casey, I. Naydenova, E. Mihaylova, A comparative cytotoxic evaluation of acrylamide and diacetone acrylamide to investigate their suitability for holographic photopolymer formulations, Int. J. Polym. Sci. 2013 (2013) 6, http://dx.doi.org/10.1155/2013/564319. Article ID 564319.

[4] M. Ortuno, E. Fernandez, S. Gallego, A. Belendez, I. Pascual, New photopolymer holographic recording material with sustainable design, Opt. Express 15 (2007) 12426-12435.

[5] M. Ortuno, S. Gallego, A. Marquez, C. Neipp, I. Pascual, A. Belendez, Biophotopol: a sustainable photopolymer for holographic data storage applications, Materials 5 (2012) 772-783.

[6] A. Olivares-Perez, M.P. Hernandez-Garnay, I. Fuentes-Tapia, J.C. Ibarra-Torres, Holograms in polyvinyl alcohol photosensitized with $\mathrm{CuCl}_{2}\left(\mathrm{H}_{2} \mathrm{O}\right)$, Opt. Eng. 50 (2011) 0658011-0658016.

[7] A. Olivares-Pérez, S. Toxqui-López, A.L. Padilla-Velasco, Nopal cactus (opuntia ficus-indica) as a holographic material, Materials 5 (2012) 2383-2402.

[8] D. Cody, E. Mihaylova, L. O'Neill, T. Babeva, H. Awala, R. Retoux, S. Mintova, I. Naydenova, Effect of zeolite nanoparticles on the optical properties of diacetone acrylamide-based photopolymer, Opt. Mater. 37 (2014) 181-187.

[9] M. Zawadzka, T. Mikulchyk, D. Cody, S. Martin, E. Mihaylova, A.K. Yetisen, J.L. Martinez-Hurtado, H. Butt, H. Awala, S. Mintova, S.H. Yun, I. Naydenova, Materials for holographic sensing, in: M. Serpe, Y. Kang, Q.M. Zhang (Eds.), Photonic Materials for Sensing, Biosensing, and Display Devices, Springer International, 2015 (in press).

[10] T. Mikulchyk, J. Walshe, D. Cody, S. Martin I. Naydenova, Humidity and temperature response of photopolymer-based holographic gratings, in: Proc. SPIE 9508, Holography: Advances and Modern Trends IV, 13-16 April 2015 Prague, Chez Republic, 2015.

[11] R. Jallapuram, I. Naydenova, H. Byrne, S. Martin, R. Howard, V. Toal, Raman spectroscopy for the characterization of the polymerisation rate in an acrylamide-based photopolymer, Appl. Opt. 47 (2) (2008) 206-212.

[12] I. Naydenova, R. Jallapuram, S. Martin, R. Howard, V. Toal, Investigations of the diffusion processes in self-processing acrylamide-based photopolymer system. Appl. Opt. 43 (14) (2004) 2900.

[13] S. Martin, I. Naydenova, V. Toal, R. Jallapuram, R. Howard, Two way diffusion model for the recording mechanism in a self developing dry acrylamide photopolymer, in: SPIE proceedings of the International Conference on
Holography, Optical Recording and Processing of Information, vol. 6252, 2006, pp. 37-44.

[14] T. Babeva, I. Naydenova, D. Mackey, S. Martin, V. Toal, Two way diffusion model for short-exposure holographic grating formation in acrylamide-based photopolymer, J. Opt. Soc. Am. B 27 (2) (2010) 197-203.

[15] M.X. Tang, A. Redler, D. Topgaard, C. Schmidt, H.S. Kitzerow, Kinetics of the grating formation in holographic polymer-dispersed liquid crystals: NMR measurement of diffusion coefficients, Colloid Polym. Sci. 290 (8) (2012) 751755.

[16] S. Gallego, A. Marquez, M. Ortuno, S. Marini, I. Pascual, A. Belendez, Monomer diffusion in sustainable photopolymers for diffractive optics applications, Opt. Mat. 33 (11) (2011) 1626-1629.

[17] D. Yu, H. Liu, Y. Jiang, X.D. Sun, Mutual diffusion dynamics with nonlocal response in $\mathrm{SiO}_{2}$ nanoparticles dispersed in PQ-PMMA bulk photopolymer, Opt. Express 19 (15) (2011) 13787-13792.

[18] O. Martinez-Matos, M.L. Calvo, J.A. Rodrigo, P. Cheben, F. del Monte, Diffusion study in tailored gratings recorded in photopolymer glass with high refractive index species, Appl. Phys. Lett. 91 (14) (2007) 141115, http://dx.doi.org/ $10.1063 / 1.2794792$.

[19] M. Toishi, T. Tanaka, K. Watanabe, K. Betsuyaku, Analysis of photopolymer media of holographic data storage using non-local polymerisation driven diffusion model, Jpn. J. Appl. Phys. 46 (6A) (2007) 3438-3447.

[20] V. Moreau, Y. Renotte, Y. Lion, Characterization of DuPont photopolymer: determination of kinetic parameters in a diffusion model, Appl. Opt. 41 (17) (2002) 3427-3435.

[21] G.H. Zhao, P. Mouroulis, Diffusion model of hologram formation in dry photopolymer materials, J. Mod. Opt. 41 (10) (1994) 1929-1939.

[22] J. Sheridan, M. Downey, F.T. O'Neill, Diffusion-based model of holographic grating formation in photopolymers: generalized non-local material responses, J. Opt. A - Pure Appl. Opt. 3 (6) (2001) 477-488.

[23] J.T. Sheridan, J.R. Lawrence, Nonlocal-response diffusion model of holographic recording in photopolymer, J. Opt. Soc. Am. A 17 (2000) 1108-1114.

[24] I. Aubrecht, M. Miler, I. Koudela, Recording of holographic diffraction gratings in photopolymers: theoretical modeling and real-time monitoring of grating growth, J. Mod. Opt. 45 (1998) 1465-1477.

[25] W.S. Colburn, K.A. Haines, Volume hologram formation in photopolymer materials, Appl. Opt. 10 (1971) 1636-1641.

[26] V.L. Colvin, R.G. Larson, A.L. Harris, M.L. Shilling, Quantitative model of volume hologram formation in photopolymers, J. Appl. Phys. 81 (1997) 5913-5923.

[27] J.H. Kwon, H.C. Hwang, K.C. Woo, Analysis of temporal behavior of beams diffracted by volume gratings formed in photopolymers, J. Opt. Soc. Am. B 16 (1999) 1651-1657.

[28] D.J. Gardiner, P.R. Graves, Practical Raman Spectroscopy, first ed., SpringerVerlag, 1989.

[29] R. Kohlrausch, Theorie des Elektrischen Rückstandes in der Leidener Flasche, Pogg. Ann. Phys. Chem. 91 (1854) 179-214.

[30] R.S. Anderssen, S.A. Husain, R.J. Loy, The Kohlrausch function: properties and applications, Anziam J. 45 (2004) 800-816.

[31] M. Berberan-Santos, E.N. Bodunov, B. Valeur, History of the Kohlrausch (stretched exponential) function: Focus on uncited pioneering work in luminescence, Ann. Phys. 17 (7) (2008) 460-461.

[32] P.G. de Gennes, Relaxation anomalies in linear polymer melts, Macromolecules 35 (2002) 3785-3786.

[33] R. Chen, Apparent stretched-exponential luminescence decay in crystalline solids, J. Lumin. 102-103 (2003) 510-518. 